\title{
Use of multicomponent seismic data for oil-water discrimination in fractured reservoirs
}

Zhongping Qian, Mark Chapman, and Xiang-Yang Li

Edinburgh Anisotropy Project, British Geological Survey.

Yonggang Zhang and Yanguang Wang

Sinopec Ltd.

Geophysicists have devoted great efforts to the problem of determining fluid saturation from seismic measurements, with some notable successes. It is commonly believed that fluid information is to be found in the P-wave data, with shear-waves being insensitive to fluid, and indeed almost all successful fluid-detection methodologies have been based on analysis of the P-wave.

Nevertheless, when we deal with fractured reservoirs, we are faced with the phenomenon of seismic anisotropy, and the rock physics relationships relevant to fractured, anisotropic, rock are more subtle than those for the more familiar isotropic case. In particular, shear-wave splitting occurs, and is known to be sensitive to the fracture properties and fluid bulk modulus. It has long been hoped that analysis of shear-wave splitting in mulitcomponent data would be able to improve our ability to detect fluids.

More recent theoretical advances in the area of frequency-dependent anisotropy have offered a new approach to this problem. These theories allow anisotropic dispersion and attenuation to be related to rock and fluid properties, typically through a fluid mobility parameter, defined as the ratio of permeability to fluid viscosity.

The ability to detect a viscosity effect is of great potential relevance to the problem of oil-water discrimination. Oil and water have similar bulk moduli, and this fact has impeded efforts to tell the two apart from analysis of seismic data. The two fluids have markedly different viscosities, however, so if we can find a robust seismic signature of fluid viscosity we would greatly improve our chances to discriminate the two fluid saturations.

In this paper we offer a theoretical analysis of wave propagation in vertically fractured rock which exhibits frequency-dependent anisotropy. For angles of incidence typical of seismic reflection data, we show that it is the slow-shear wave which suffers most attenuation and whose properties are most sensitive to fluid viscosity. Further to this, we demonstrate that the converted-wave amplitude in the fracture normal direction can be very sensitive to the fluid, even when the P-wave attributes are insensitive to fluid.

Based on our analysis, we devise a processing strategy which is applied to 3D3C data from Shengli oilfield in China, which has undergone water flooding. We find amplitude and traveltime anomalies which correlate with known water and oil saturated zones, and which are consistent with the effects predicted by our theoretical modelling. We conclude that proper processing and interpretation of multicomponent data can help us to discriminate oil and water saturation in fractured reservoirs. 


\section{Theoretical background}

We consider fracture distributions in porous rock which are approximately aligned and vertical. Traditional techniques for modelling the elastic properties of such systems make use of static equivalent medium theories in the long wavelength limit. Such theories have many features in common, and the simplest can be shown to be consistent with the notation of Schoenberg, in which the "excess compliance" associated with the fracture network can be written in terms of two parameters $Z_{N}$ and $Z_{T}$ which are the normal and shear compliances respectively of the fractures.

A typical assumption is that $Z_{T}$ would be insensitive to fluid whereas $Z_{N}$ would decrease with increasing fluid bulk modulus. Theories which assume that the cracks are penny shaped allow explicit computation of $Z_{N}$ in terms of the fluid properties, either assuming that the cracks are fluid-isolated (based on Hudson's theory) or allowing fluid communication with the surrounding rock (following Thomsen's model).

Such theories demonstrate that for near-vertical propagation, shear-wave splitting can vary markedly with fluid saturation. The predicted change in shear-wave splitting arising from a given change in fluid properties varies markedly between the different theories, but the models do predict a consistent trend. Fluids with lower bulk moduli give rise to increased $Z_{N}$ values, and this tends to lead to an increase in measured shear-wave splitting, for near vertical propagation. It is the slower quasi-shear wave whose velocity changes with the fluid; the faster wave is a pure-shear wave whose velocity is insensitive to fluid bulk modulus in accordance with Gassmann's relation.

More complex theories attempt to predict the anisotropic dispersion and attenuation which is generated by fluid-saturated fracture systems. In these theories a central role is played by the fluid mobility parameter (see Batzle et al., 2006, Geophysics):

$$
M=\frac{k}{\eta}
$$

in which $k$ is the permeability and $\eta$ is the fluid viscosity. The fluid mobility appears in a "characteristic frequency", which divides the frequency range into broadly three bands; a low frequency range, a transition band and a high frequency range. The low and high frequency ranges correspond to the static cases in which we assume fluidcommunicating or fluid-isolated cracks respectively. The behaviour in the transition band is rather different, since in this case the velocities change rapidly with frequency and attenuation occurs.

In the case in which we have a single vertical fracture set, theoretical models predict that the attenuation should be strongly anisotropic. Waves propagating in the plane of the fractures are predicted to suffer very little attenuation, but in the plane perpendicular to the fractures strong attenuation can take place.

Figure 1 demonstrates typical behaviour, assuming propagation perpendicular to the fractures, for all three wave modes as a function of polar angle (see Chapman, 2003, 
Geophysical Prospecting for details of the theory). We set the convention that for a vertical fracture set, a polar angle of 0 degrees corresponds to vertical propagation while 90 degrees is the horizontal. The pure shear-wave should suffer no fluid related attenuation, but both the P-wave and quasi-shear wave can be strongly attenuated.

This behaviour comes about because the attenuation is assumed to occur due to the relaxation of fluid-pressure gradients between the fractures and the surrounding porespace. The pure-shear wave does not compress either the fractures or the pore-space and so can create no such attenuation. Both the P-wave and quasi-shear do compress the fractures relative to the pore-space and create attenuation. The effect is most pronounced for the P-wave, which suffers the highest absolute attenuation of any of the waves when it propagates at 90 degrees. Nevertheless the polarisations of the Pand quasi-shear are at right angles to one another, and this means that the angle of maximum attenuation differs for the two modes. In particular, while the P-wave has maximum attenuation for horizontal propagation the maximum quasi-shear attenuation occurs for propagation around 45 degrees.

In reflection seismology we are typically concerned with angles of incidence between around 0 and 45 degrees. A striking conclusion to be drawn from Figure 1 is that for such angles of incidence, the greatest effect of the fracture related attenuation should be on the quasi-shear wave. Furthermore, for these angles of incidence the quasi-shear mode is typically the slow shear-wave.

The strong attenuation of the slow shear-wave has many implications, but in this study we focus on the implications for fluid-substitution. For each wave-mode and for each direction of propagation, the behaviour is predicted to resemble that of a standard linear solid. In particular, the wave propagation exhibits the Kramers-Kronig relationship between attenuation and dispersion.

Figure 2 shows the predicted velocities as a function of non-dimensional frequency corresponding to the model discussed above for all wave-modes propagating at 30 degrees from vertical and for two different viscosities. Figure 2a demonstrates the predicted P-wave behaviour. The velocities increase with frequency, but the change is modest since there is little attenuation for this direction. When the viscosity is increased the dispersion curve shifts to lower frequencies, creating a frequency band in which the velocity is sensitive to viscosity. Figure $2 b$ shows the corresponding shear-wave behaviour. The faster shear-wave is not attenuated, and so its velocity does not depend on viscosity. The slow-shear wave suffers more attenuation than the $\mathrm{P}$-wave and this corresponds to a greater frequency dependence of the velocities. This leads to a frequency band in which the slow shear-wave is very sensitive to the viscosity.

Substituting one fluid for another involves changing at least the fluid bulk modulus, viscosity and density. In the water-to-oil case, the oil typically has a slightly lower bulk modulus and density than the water, but a much larger viscosity. Fluidsubstitution theories which ignore the viscosity typically suggest that oil-water discrimination is practically impossible. Since we expect theoretically that the slow shear-wave should be dependent on viscosity in fractured media, it is worth considering whether this effect can be used for oil-water discrimination in fractured reservoirs. 
Certainly, shear-wave splitting is sensitive to oil-water substitution in the modelling framework we describe. Figure 3 shows shear-wave splitting as function of frequency for oil and water saturation. In the low-frequency limit oil saturation gives rise to higher values of shear-wave splitting, since the lower oil bulk modulus makes the fractures more compliant than the water-saturated case. In the high frequency limit there is little effect of the fluid for thin cracks, although oil will give higher values of shear-wave splitting if we assume much fatter cracks. Between these cases we have again a transition zone corresponding to the frequency dependence of the slow-shear velocity as shown in Figure 2. We note that within the transition zone the sensitivity to fluid is greater than, and the direction of the change opposite to, that predicted in either low or high frequency limit.

This behaviour has implications for converted wave reflectivity. We demonstrate this effect with a numerical example, in which the parameters have been taken from velocity logs from Shengli Oilfield, seismic data from which is to be discussed below. The model which we consider consists of a high-to-low interface which has an isotropic layer overlying a vertically fractured target. In the general case, an incident P-wave will generate 3 reflections corresponding to the PP, PS1 and PS2 modes. If we confine ourselves to propagation in the symmetry planes however (parallel and perpendicular to the fracture strike), only 2 reflections, one PP and another PS, will be generated.

Figure 4 shows the PP and PS reflection coefficients for waves propagating parallel and perpendicular to the fractures, for both water and oil saturation. As expected, for the PP reflection there is very little sensitivity to the fluid in either direction. This is because of the low contrast in bulk modulus between oil and water and little viscosity dependence for these angles of incidence; indeed the bulk modulus and viscosity effects even tend to cancel each other out. Likewise, when we assume propagation parallel to the fracture strike the PS amplitude is predicted to be insensitive to the saturating fluid. This is not the case for propagation perpendicular to the fracture strike, however. In that case the PS reflection is sensitive to the saturating fluid. This is because of the effect of the fluid viscosity for shear-wave propagation in that direction.

To further demonstrate the behaviour we calculate synthetic seismograms for a simple single layer model, with an isotropic layer overlying a fractured layer with horizontal transverse isotropy, where the angle of incidence is taken to be up to 45 degrees. Sample synthetic seismograms are shown in Figure 5.

We find that the converted wave amplitude is indeed sensitive to the saturating fluid. Figure 6 shows the difference plots for PP and PS reflections parallel and perpendicular to the fractures. A strong anomaly is present for the converted wave travelling perpendicular to the fractures. No anomaly is present for the P-wave in either direction, or for the converted wave parallel to the fractures. To make this effect clear, we pick the amplitudes of the reflections for the different modes and directions and display them in Figure 7. Only for the converted wave travelling perpendicular to the fractures is there fluid-dependence of the amplitudes, with water saturation acting to dim the converted wave amplitude in that case. 
Recent observations of frequency-dependent shear-wave splitting in VSP and microseismic data suggest that such effects may well be important at seismic frequencies, as is theoretically predicted when we have "meso-scale" fractures, but it remains an open question to demonstrate whether viscosity effects can be detected in seismic data. In the remainder of this paper we consider a multicomponent dataset from Shengli oilfield in China, and argue that such effects can indeed be detected in the data.

\section{Field background and seismic data processing}

In this paper we consider 3D3C data from the Ken 71 area of the Shengli Oilfield. This is a 20 square-kilometre zone located at the Yellow River delta beside the Bohai Sea. The area is relatively flat, and the reservoir is a gentle anticline with heavy faulting. The reservoir has undergone production through water-flooding, which has altered the fluid composition and pressure.

In 2005 an integrated programme of reservoir geophysics was implemented, aiming to characterize the bypassed oil in the area. This included the acquisition of high resolution 3D3C seismic data, together with cores, logs, 3D VSP's and crosswell seismic. Digital MEMS sensors were used to improve data quality for the acquisition of the 3D3C data.

Processing of the converted-wave data faced two main challenges: the asymmetric raypath of the converted-wave and the effect of vertical transverse isotropy (VTI) in the background sand-shale sequence, which gives rise to strong non-hyperbolic moveout in the pre-stack data. To address these problems we designed a two-stage work flow.

In the first stage we determine the principal coordinate system, or the polarisation directions of the fast and slow shear wave. The main steps are coordinate rotation, noise reduction and statics, followed by azimuthal analysis of the transverse component data. This azimuthal analysis aims to identify the principal directions by identifying polarity reversals. In this dataset, the fast direction was identified as N45E, which was in agreement with the structural alignment of the area.

In the second stage we account for the VTI effects and asymmetric raypath whilst preserving shear-wave splitting. We achieve this by dividing the data into different azimuthal sectors and then processing each azimuth separately. Using a 3 parameter theory which accounts for both the VTI and asymmetric raypath effects (see Li and Yuan, 2003, for details). In this case, the data are divided into two orthogonal sectors (45 and 135 degrees), and high quality fast and slow shear-wave volumes are obtained.

\section{Data analysis and interpretation}

Our analysis of the data has two components. We begin by considering shear-wave splitting through the computation of time delay gradient sections, before studying the azimuthal variation of the converted wave amplitude. 
We used a short time-window correlation method to create time delay spectra, which can then be differenced to create a time-delay gradient section. Such a section is shown in Figure 8. A time delay anomaly is clearly visible around well J-41, where water injection has taken place. In general, we find that water saturated zones tend to have higher values of shear-wave splitting, whereas zones where the original oil is in place tend to exhibit low values of shear-wave splitting. The difference is rather large, with around an extra 5\% shear-wave anisotropy in the water saturated zones.

Comparison of the PP and PS sections for the fast and slow directions reveals amplitude anomalies for the slow PS section which appear to correlate with fluid saturation. Figure 9 shows that the P-wave sections from the two directions show little differences, but that the amplitude of the slow PS section appears to be dimmer in the water saturated zones.

We now consider in detail the differences between converted-wave data from two well locations, one known to be in an oil saturated zone and the other from a water saturated zone. Figure 10 shows that for the water saturated zone we have a dim amplitude, with little difference between the fast and slow directions. The oil saturated well corresponds to higher amplitude, but this time there is a significant difference between the fast and slow azimuth. The slow azimuth has a much stronger amplitude than the fast azimuth. This is entirely consistent with our theoretical modelling.

\section{Conclusions}

In this paper we have demonstrated theoretically that seismic measurements of the properties of the slow shear-wave can potentially give access to fluid saturation information in fractured rock, even in circumstances in which the P-wave response is rather insensitive to fluid. This is possible because for angles of incidence between 0 and 45 degrees the slow shear-wave is strongly attenuated and dispersed, in contrast to the P-wave and fast shear-wave which suffer little fracture-related attenuation in these directions. For the slow shear-wave propagating near-vertically, the main effect of fluid-substitution comes through the change in fluid viscosity.

We analyse 3D3C data from Shengli oilfield and detect amplitude and travel-time anomalies which are consistent with our theoretical modelling and which can be correlated with well information on the fluid saturation. Water saturated zones tend to be associated with higher values of shear-wave splitting than oil saturated zones, and their amplitudes are dimmer. We note that this is the exact opposite of what would be expected from an anisotropic fluid-substitution calculation using the change in bulk modulus alone without a viscosity component. In the oil saturated zones we see a strong change in converted wave amplitude between the fast and slow direction, while in water saturated zones there is no azimuthal change. This behaviour is predicted by our theoretical modelling.

Undoubtedly some uncertainties remain, principally related to the effect of pore-fluid pressure which presumably differs significantly between the water flooded and oil saturated zones. Nevertheless, we are encouraged by the correspondence between the shear-wave data and the fluid saturation information, and believe that the use of 
multicomponent data to discriminate oil and water saturation in fractured reservoirs will be a fruitful direction in the future.

\section{Suggested reading}

"The 3D shear experiment over the Natih field in Oman: the effect of fracture-filling fluids on shear propagation" (van der Kolk, Guest and Potters, Geophysical Prospecting 2001). "Frequency-dependent anisotropy due to meso-scale fractures in the presence of equant porosity" (Chapman, Geophysical Prospecting, 2003). "Processing, modelling and predicting time-lapse effect of overpressured fluidinjection in a fractured reservoir" (Angerer, Crampin, Li and Davis, 2002). "Converted-wave moveout and conversion-point equations in layered VTI media: theory and application" (Li and Juan, 20003, Journal of Applied Geophysics). "Fluid mobility and frequency-dependent seismic velocity - Direct Measurements" (Batzle, Han and Hoffman, Geophysics, 2006). "Fluid-dependent shear-wave splitting in a poroelastic medium with conjugate fracture sets" (Galvin, Gurevich and Sayers, Geophysical Prospecting, 2007)

\section{Acknowledgements}

We thank SinoPec Shengli Oilfield for permission to show the data. We would also like to thank Lifeng Wang for his help with the data analysis, and in particular for preparing Figure 8. This work is supported by the SinoPec international collaboration programme and SinoPec Shengli Oilfield Ltd through the Edinburgh Anisotropy Project of the British Geological Survey (BGS), and is published with the approval of all project partners and the Executive Director of the BGS. 


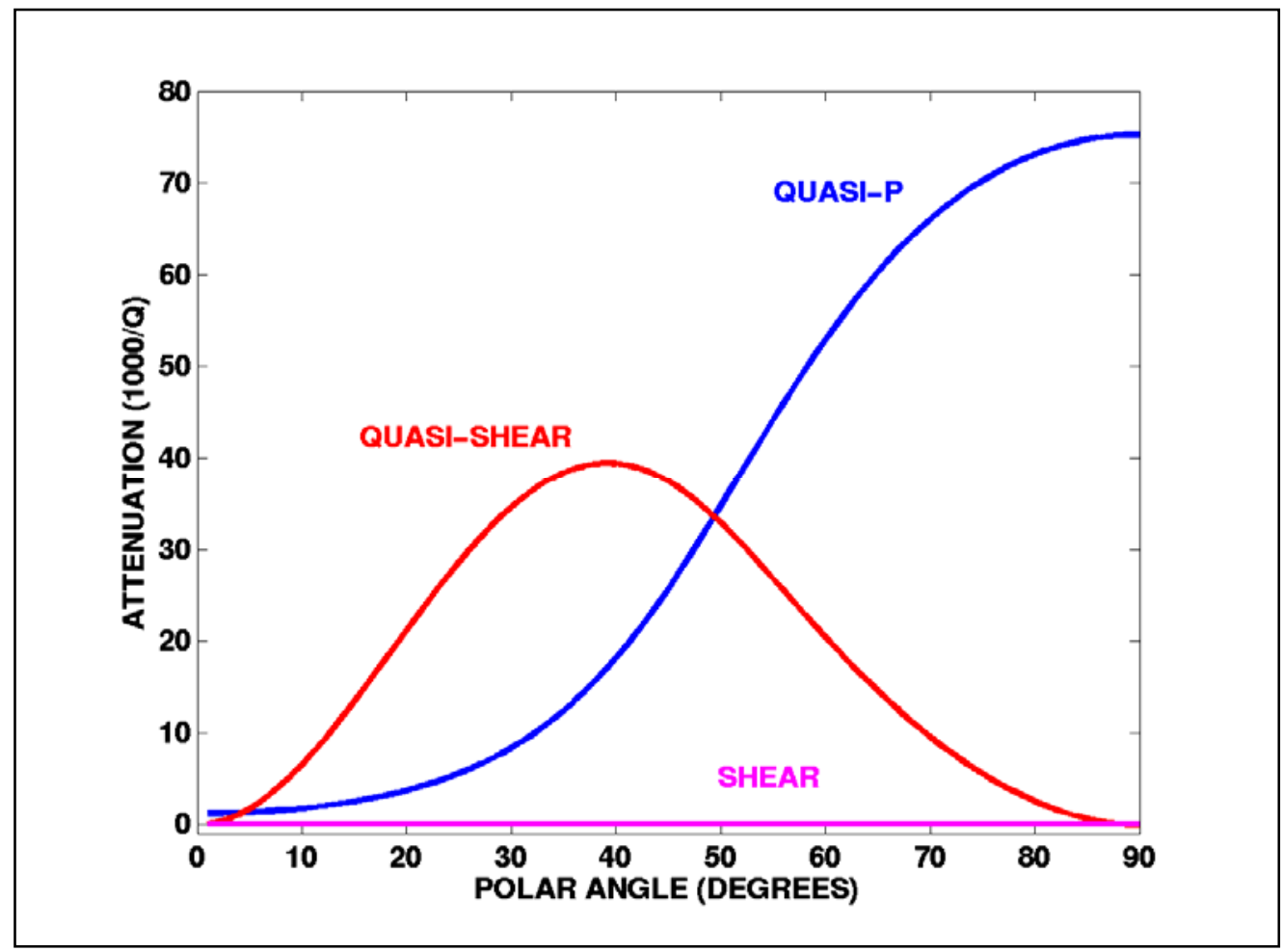

Figure 1: Calculated attenuation for the 3 wave modes as a function of polar angle ( 0 degrees is vertical, 90 degrees horizontal) for propagation perpendicular to the fractures. 

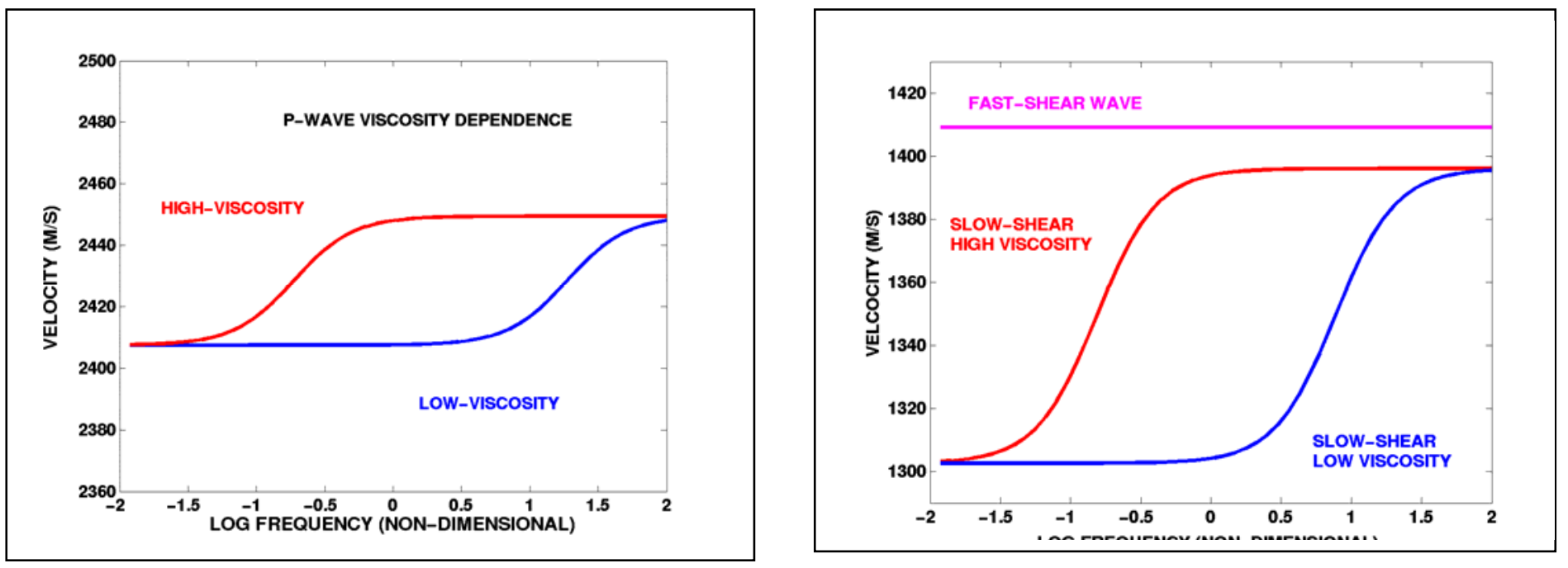

Figure 2: Velocity as a function of non-dimensional frequency for P-wave and fast and slow shearwaves, for two different viscosities. Propagation is perpendicular to the fractures, with a polar angle of 30 degrees. 


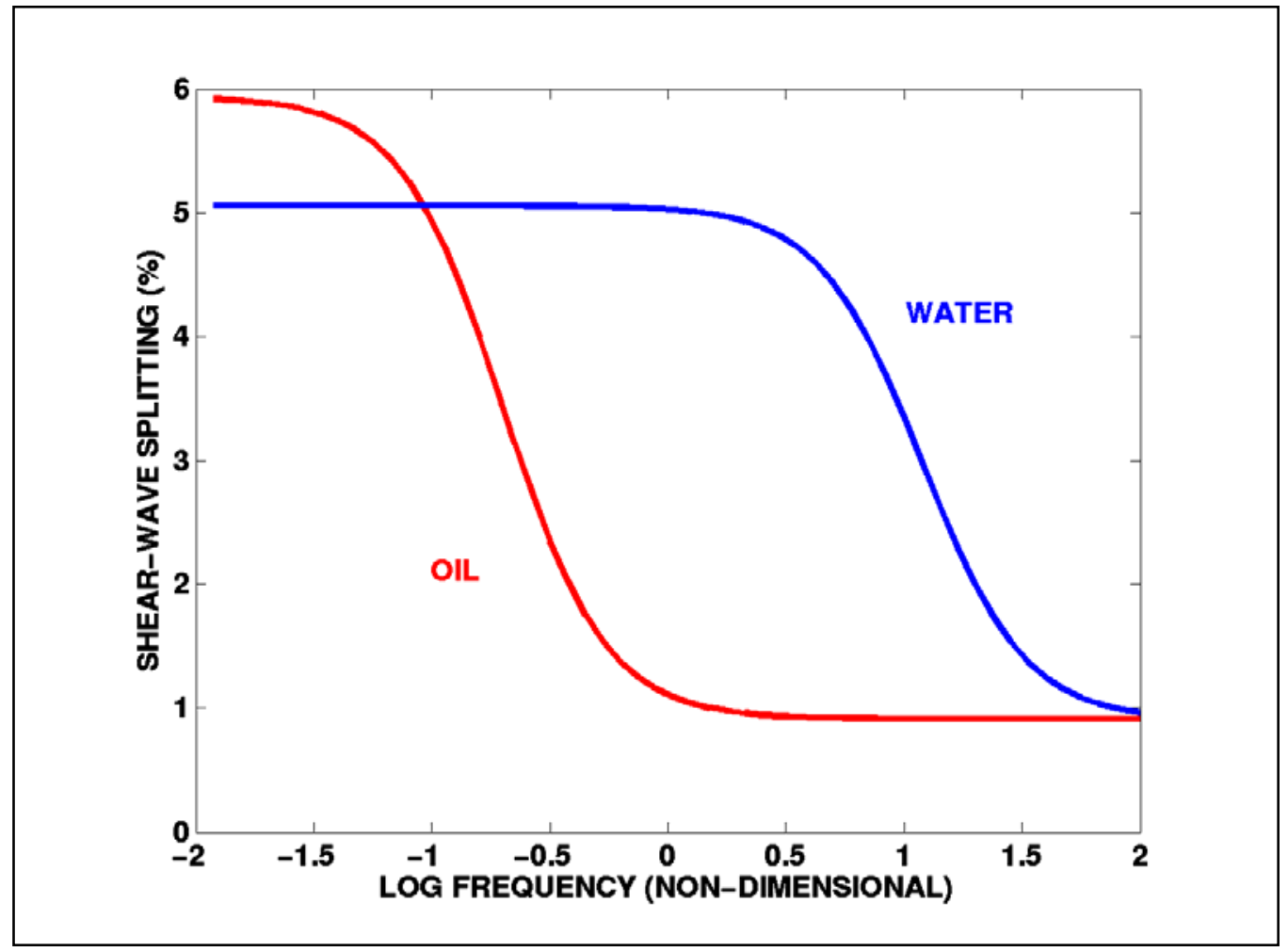

Figure 3: Predicted values of shear-wave splitting as a function of frequency for oil and water saturation. 

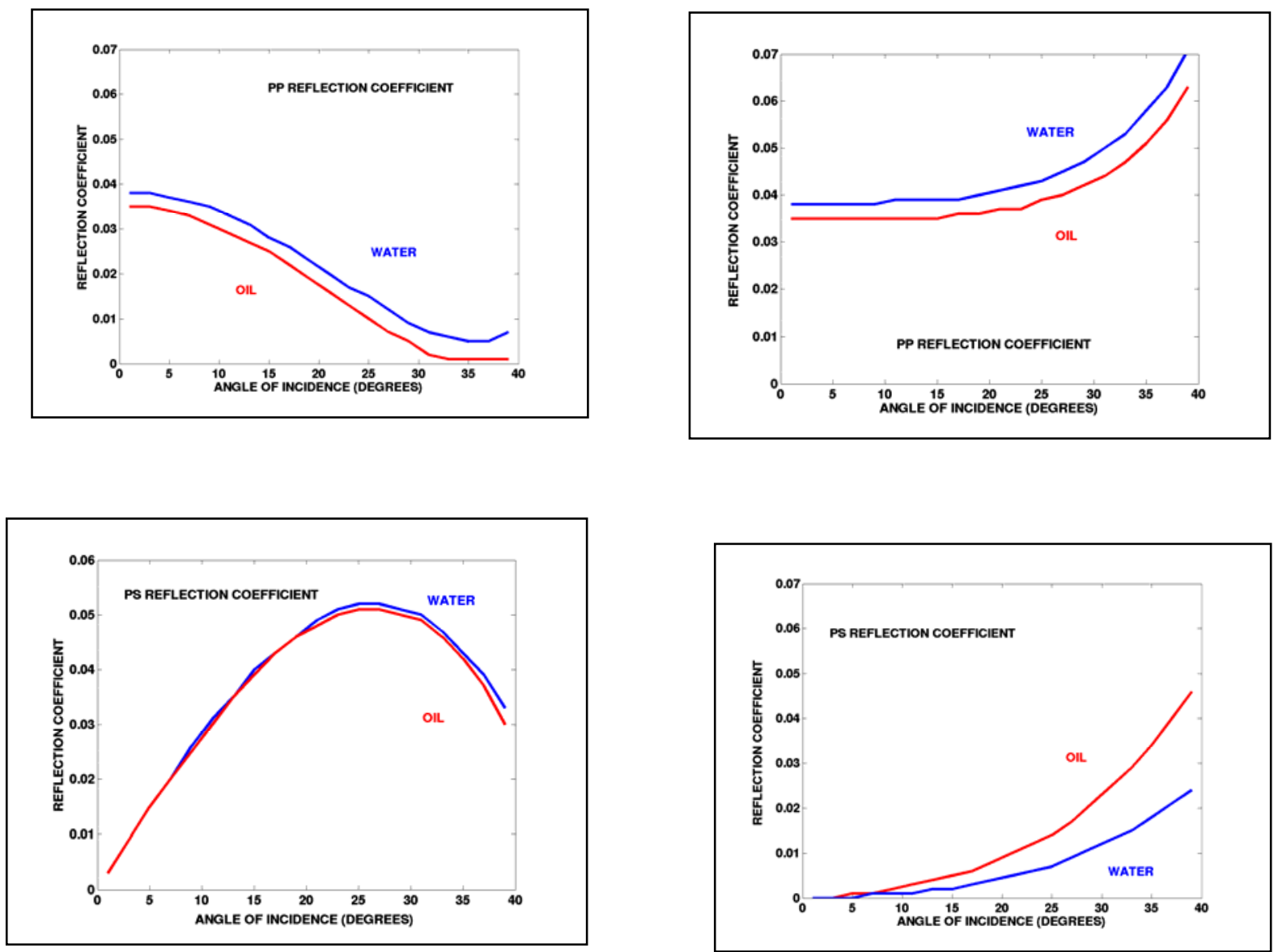

Figure 4: Computed values for the reflection coefficient. Top diagrams are for PP, bottom for PS. Left diagrams are parallel to the fractures, right are perpendicular. 


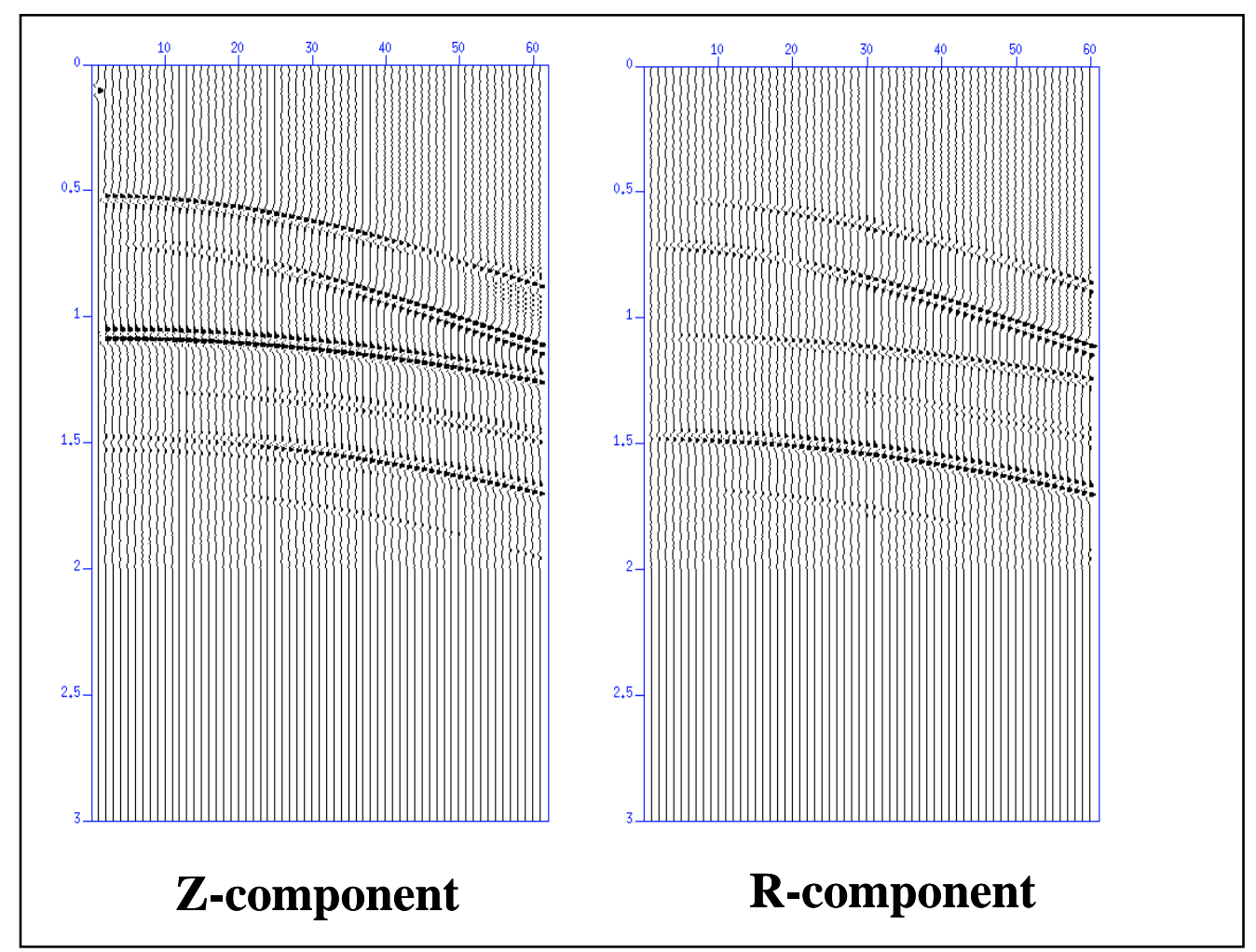

Figure 5: Sample synthetic seismograms for our model of a fractured layer, propagation is parallel to the fractures. 

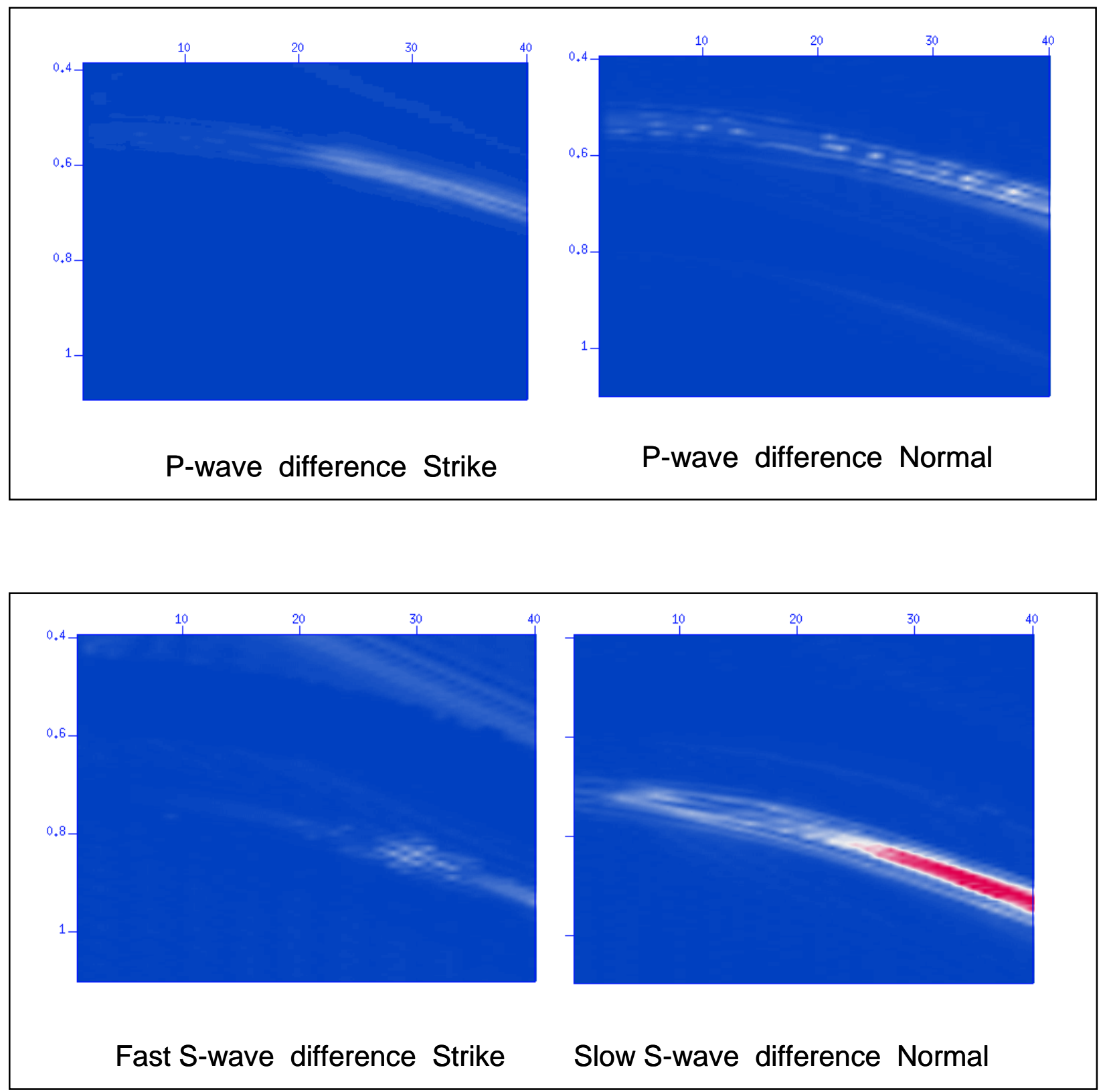

Figure 6: Results of differencing the synthetics calculated for oil saturation from those calculated for water saturation. Top is P-wave, bottom the converted wave. Left diagrams are for propagation in the fracture strike direction, right are in the fracture normal direction. Only the converted wave propagating normal to the fractures shows sensitivity to fluid. 

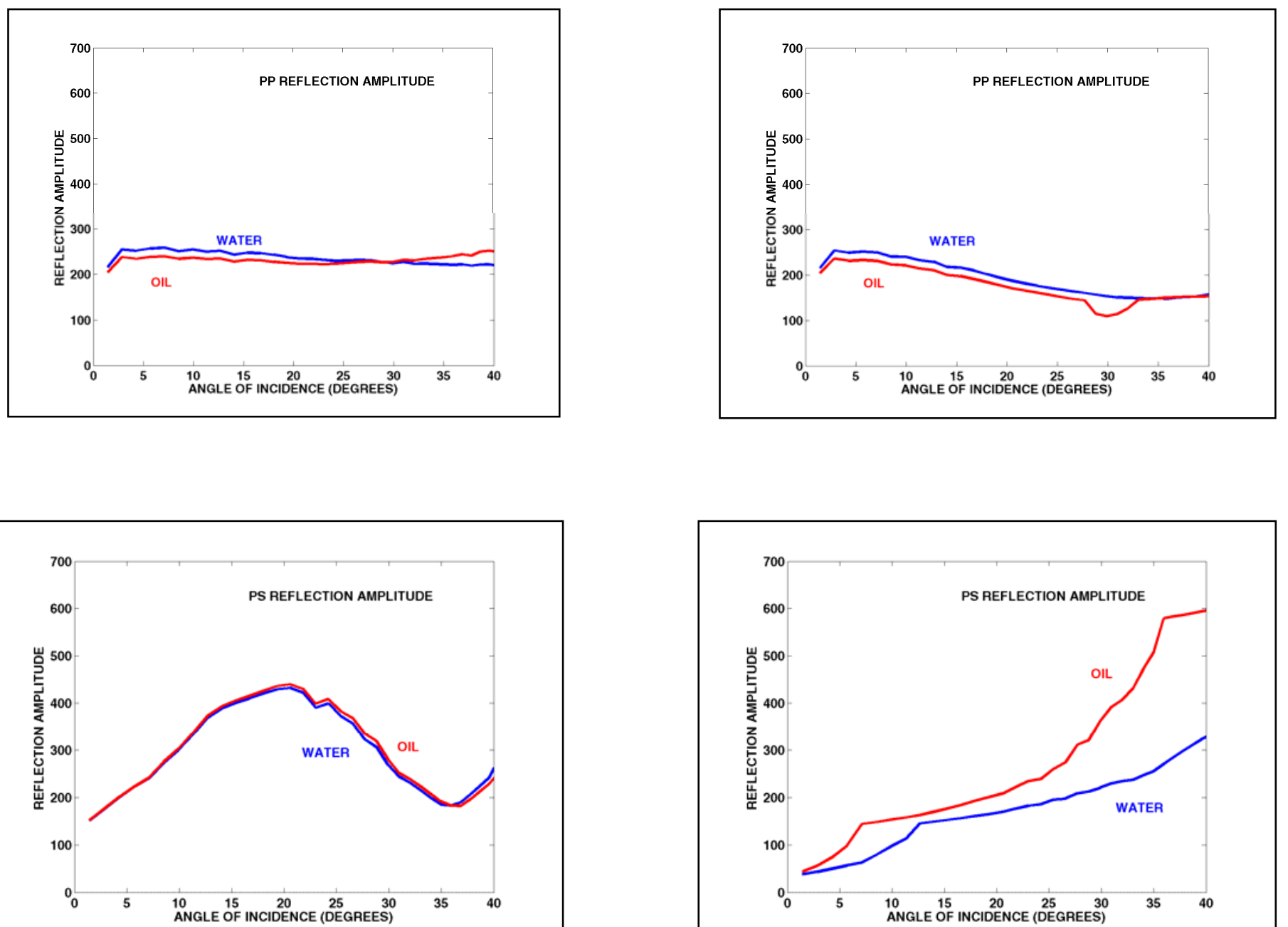

Figure 7: Picked amplitudes from the synthetic seismograms under oil and water saturation. Top diagrams are PP, bottom PS. Left diagrams are for propagation parallel to the fractures, right for propagation in the fracture normal direction. 


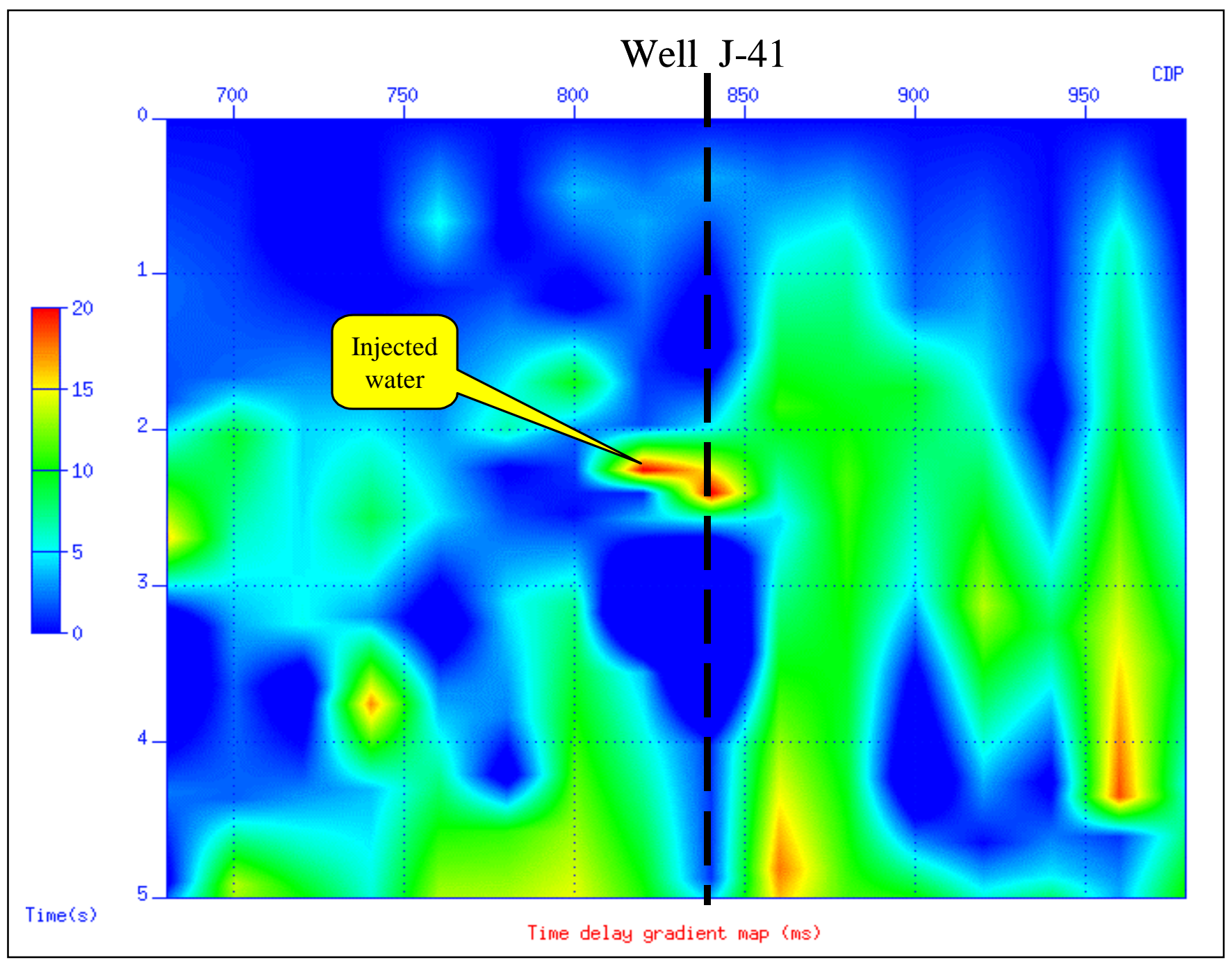

Figure 8: Time delay gradient section. The indicated anomaly is the zone at which water injection has taken place. 


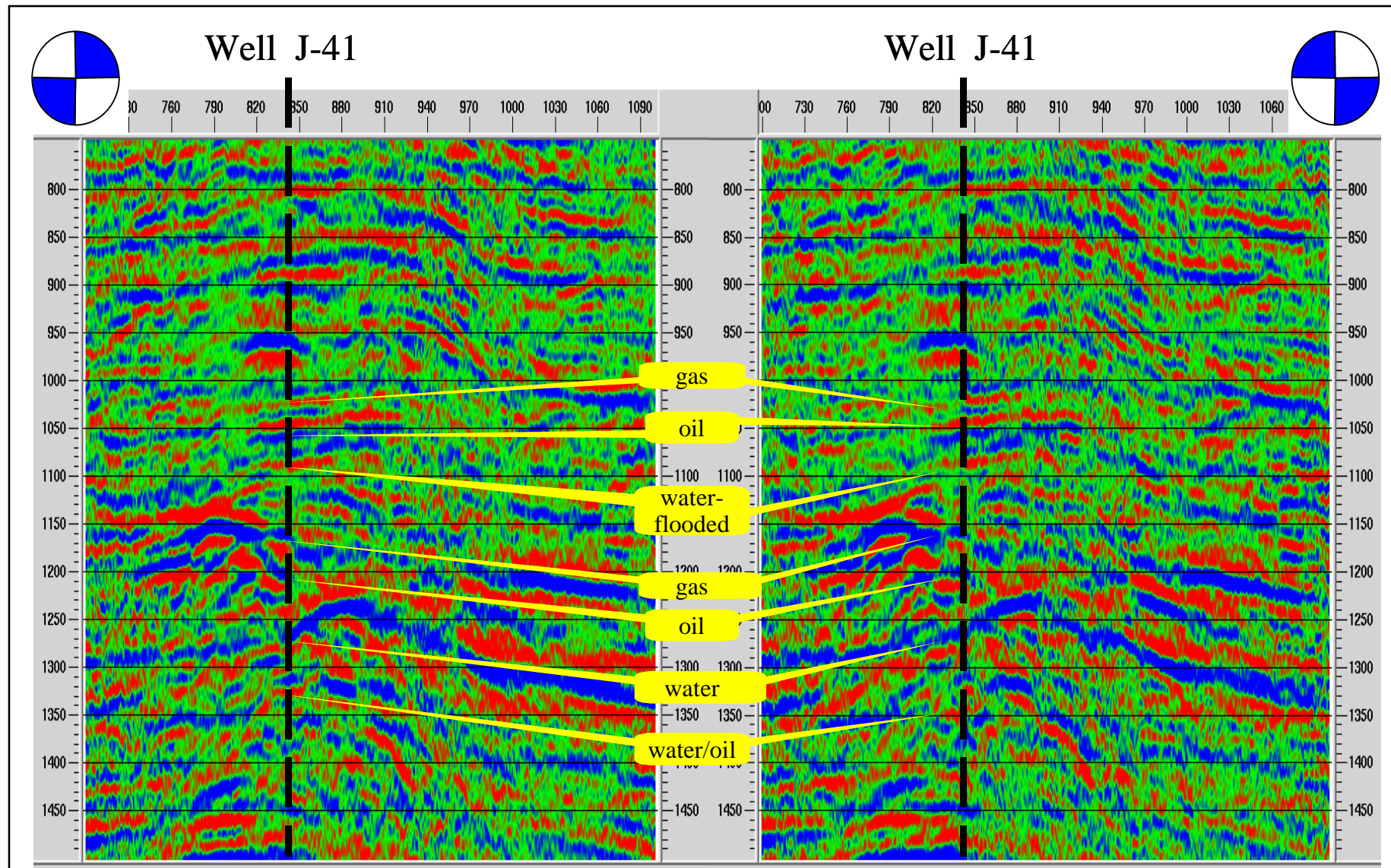

Z-component $45^{\circ}$

Z-component $135^{\circ}$

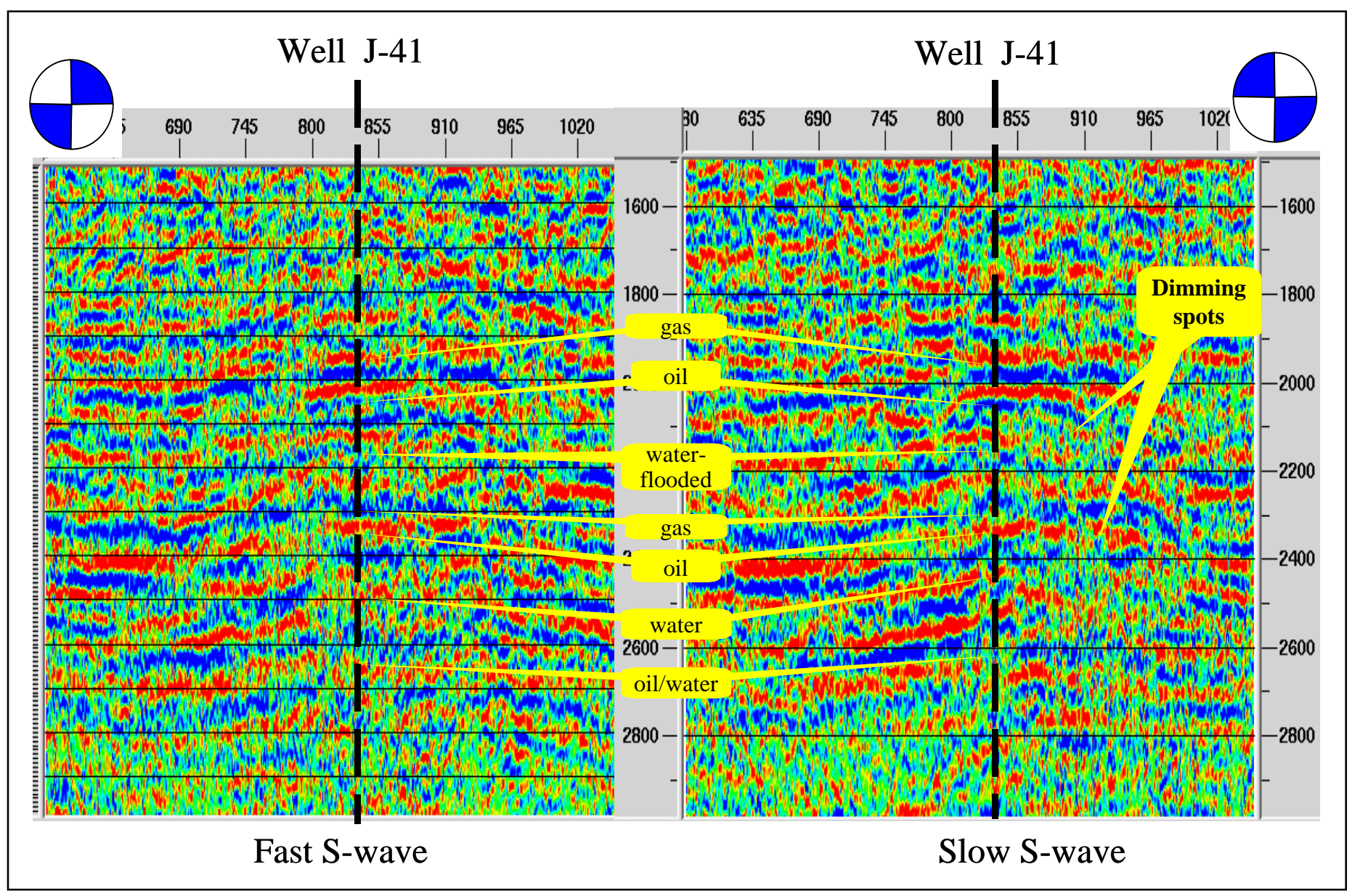


Figure 9: Stacked sections for PP (top) and PS (bottom) created from data sectored from the fast (left) and slow (right) directions. Notice the similarity between the PP sections, while the slow PS section appears to show fluid-related amplitude effects. 


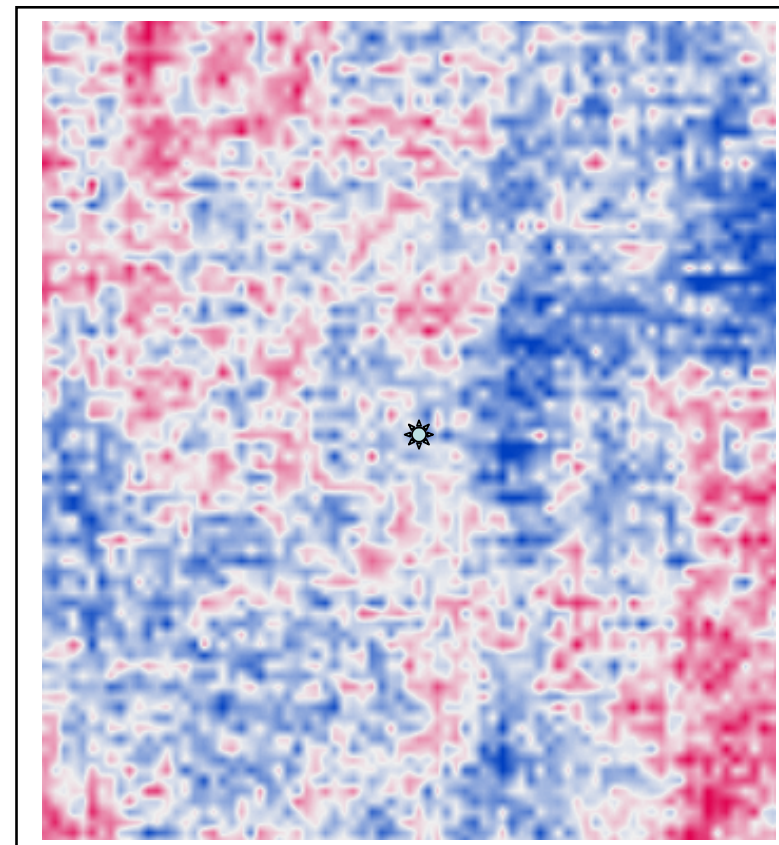

Water bearing layer Fast S-wave

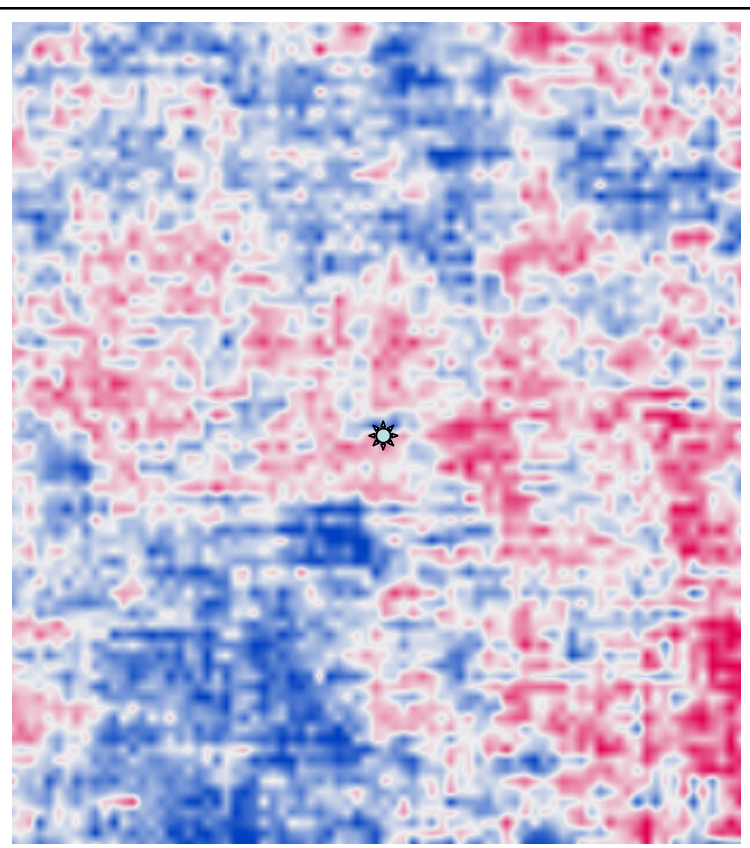

Water bearing layer Slow S-wave slice

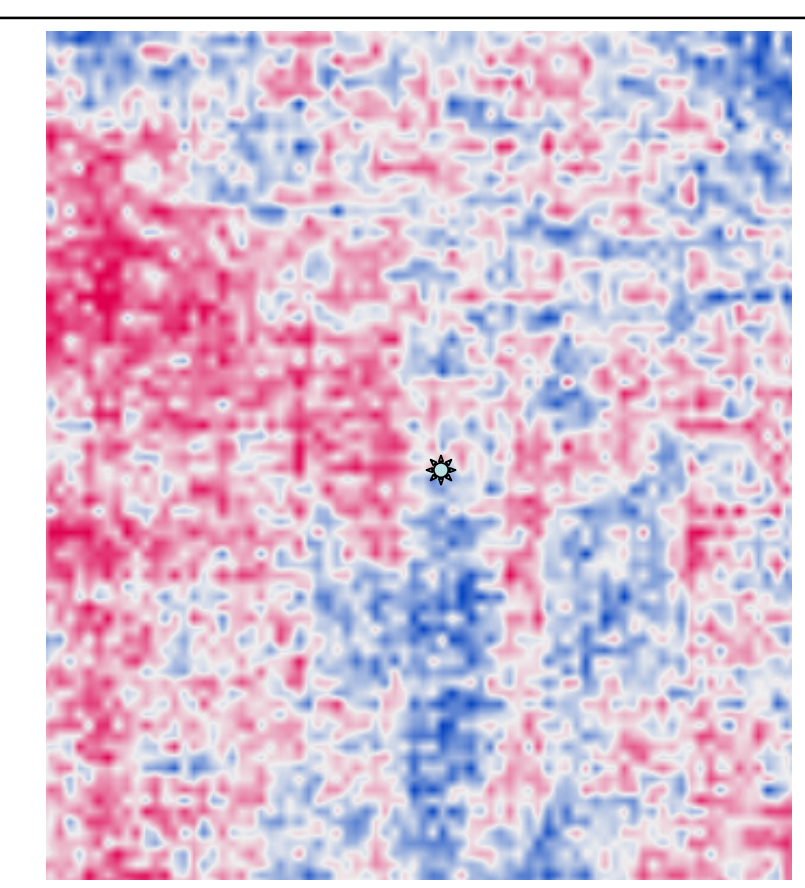

Oil bearing layering Fast S-wave slice

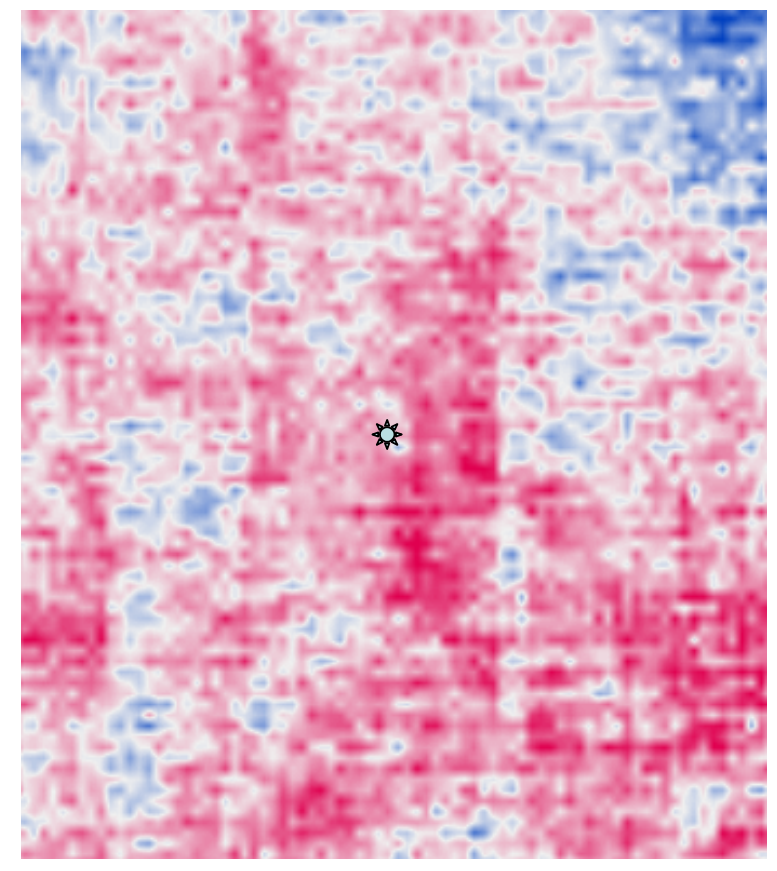

Oil bearing layer Slow S-wave slice 
Figure 10: Horizontal amplitude slice from converted wave sections around wells in water saturated zone (top) and oil saturated zone (bottom). Left is the fast direction, right is the slow direction. Note the difference in amplitude between the fast and slow direction amplitude in the oil saturated case, compared to the similarity between the two directions in the water saturated case. 